\title{
Produção e estabilidade de conservação de farinha de acerola desidratada em diferentes temperaturas
}

\author{
Production and storage stability of acerola flour dehydrated at different temperatures
}

\author{
Daíse Souza Reis ${ }^{*}$, Acácio Figueiredo Neto $^{2}$, Andréa de Vasconcelos Ferraz ${ }^{3}$, Sérgio Tonetto de Freitas ${ }^{4}$ \\ 1 Universidade Federal do Vale do São Francisco (UNIVASF), Programa de Pós-graduação em Engenharia Agrícola, Juazeiro/BA - Brasil \\ 2 Universidade Federal do Vale do São Francisco (UNIVASF), Colegiado de Engenharia Agrícola, Juazeiro/BA - Brasil \\ ${ }^{3}$ Universidade Federal do Vale do São Francisco (UNIVASF), Colegiado de Engenharia Elétrica, Juazeiro/BA - Brasil \\ ${ }^{4}$ Empresa Brasileira de Pesquisa Agropecuária (Embrapa), Embrapa Semiárido, Petrolina/PE - Brasil
}

\section{*Corresponding Author}

Daíse Souza Reis, Universidade Federal do Vale do São Francisco (UNIVASF), Colegiado de Engenharia Agrícola e Ambiental, Avenida Antônio Carlos Magalhães, 510, Country Club, CEP: 48902-300, Juazeiro/BA - Brasil, e-mail: dayse29@hotmail.com

Cite as: Production and storage stability of acerola flour dehydrated at different temperatures. Braz. J. Food Technol., v. 20, e2015083, 2017.

Received: Oct. 28, 2015; Accepted: Feb. 15, 2017

\section{Resumo}

O objetivo deste trabalho foi identificar a melhor temperatura de desidratação para a produção de farinha de acerola de alta qualidade de consumo e estabilidade de parâmetros físico-químicos durante a conservação. Frutos de acerola 'Flor Branca' foram colhidos no estádio de maturação maduro em um pomar comercial do Vale do São Francisco, Petrolina, PE. Frutos com ou sem sementes foram sanitizados e desidratados nas temperaturas de $60{ }^{\circ} \mathrm{C}, 70{ }^{\circ} \mathrm{C}$ e $80^{\circ} \mathrm{C}$ em estufa de circulação de ar forçado e, em seguida, foram triturados para a obtenção da farinha, a qual foi armazenada em temperatura ambiente em embalagens de polietileno herméticas. A polpa dos frutos foi utilizada para a determinação do pH, sólidos solúveis (SS), acidez titulável (AT), ácido ascórbico (AA) e umidade. A farinha de acerola foi avaliada a cada 15 dias, por um período de 75 dias, quanto ao $\mathrm{pH}, \mathrm{SS}, \mathrm{AT}, \mathrm{AA}$ e cor. Para todas as temperaturas de desidratação, os valores de $\mathrm{pH}$ e SS foram semelhantes, variando entre 3,5-4,2 e 6,3-11,7, respectivamente. Os SS apresentaram menor variação ao final do armazenamento. A AT aumentou em todas as amostras variando de $5 \%$ a 10\%. As perdas de ácido ascórbico foram de $76,2 \%$ e $80 \%$, $23,9 \%$ e $55 \%$, ou $37,9 \%$ e $65 \%$ para as farinhas com e sem sementes desidratadas a $60{ }^{\circ} \mathrm{C}, 70{ }^{\circ} \mathrm{C}$ e $80{ }^{\circ} \mathrm{C}$, respectivamente. De acordo com os resultados obtidos, a melhor temperatura de desidratação é $70{ }^{\circ} \mathrm{C}$, pois resultou em farinha com alta qualidade de consumo e estabilidade de parâmetros físico-químicos durante 75 dias de conservação.

Palavras-chave: Frutos; Secagem; Vida de prateleira; Ácido ascórbico.

\section{Abstract}

The objective of this work was to identify the best dehydration temperature for the production of high quality acerola flour and to provide stability of the physicochemical parameters during storage. 'Flor Branca' acerolas were harvested at the red ripe stage in a commercial orchard in the São Francisco Valley, Petrolina, PE, Brazil. Fruits with or without seeds were dried at 60,70 and $80^{\circ} \mathrm{C}$ in a forced air circulating oven. The dried fruits were then ground to obtain the flour, which was stored at room temperature in hermetically sealed polyethylene bags. The fresh fruit pulp was evaluated for its $\mathrm{pH}$ value, soluble solids (SS), titratable acidity (TA), ascorbic acid (AA) and moisture contents. The acerola flour was analysed every 15 days for 75 days for $\mathrm{pH}$, SS, AT, AA and colour. For all the drying temperatures, the $\mathrm{pH}$ and SS showed similar levels, ranging from 3.5 to 4.2 and 6.3 to 11.7, respectively. The TA increased during storage for all the drying temperatures, ranging from 5 to $10 \%$. The AA losses were 76.2 and $80 \%, 23.9$ and $55 \%$, and 37.9 and $65 \%$ for flours obtained from fruits with and without seeds, dehydrated at 60,70 and $80^{\circ} \mathrm{C}$, respectively. According to the results, the best drying temperature was $70^{\circ} \mathrm{C}$, which resulted in high flour quality and physicochemical stability during the 75 days of storage.

Keywords: Fruits; Drying; Shelf life; Ascorbic acid. 


\section{Introdução}

A acerola é um fruto de grande valor nutricional por apresentar altas concentrações de ácido ascórbico $(\mathrm{AA})$, que podem chegar a valores de $5 \%$, agregando um alto potencial nutricional para o consumo do fruto fresco ou industrializado (MENDONÇA; MEDEIROS, 2011; SEGTOWICK et al., 2013). A industrialização da acerola para obtenção da farinha é uma alternativa para diversificar as possibilidades de comercialização dos frutos, o que permite aumentar a estabilidade e reduzir as perdas pós-colheita do produto, tornando possível o armazenamento em condições ambientais por maior tempo e propiciando o consumo do produto fora da safra. Estudos realizados por Menezes et al. (2009) constatam que, após a desidratação da acerola, foi possível preservar aproximadamente $67 \%$ dos conteúdos de AA no produto processado.

A farinha de acerola é um produto que pode ser obtido da desidratação da fruta na forma in natura, ou dos resíduos provenientes do processamento, como opção para resolver o problema das perdas pós-colheita causadas pela sensibilidade dos frutos, principalmente durante a colheita e transporte. Deste modo, a produção da farinha de acerola é uma das formas de garantir a conservação dessa fruta por mais tempo, e de melhor aproveitamento dos seus constituintes (CARNEIRO; MELLO, 2011).

Este subproduto pode ser um alimento para consumo direto, ou servir como aditivo de outros produtos alimentícios. Aquino et al. (2010) comprovaram a viabilidade da substituição da farinha de trigo pela farinha de resíduo de acerola na produção de biscoitos tipo cookies, resultando em um produto altamente rico em ácido ascórbico ( $A A)$, atingindo $2,17 \%$ de $A A$, além de ser rico em proteínas e lipídios.

Todos os produtos apresentam um tempo de vida útil que é caracterizado pela sua estabilidade nutricional, sensorial, química, física e microbiológica. Entende-se por estabilidade de um produto o período no qual ele pode ser consumido de forma que as suas características nutricionais e microbiológicas estejam conservadas (YUYAMA et al., 2008). Geralmente as vitaminas, ácidos, carboidratos, proteínas entre outros constituintes, são sensíveis a fatores externos e internos como a luz, temperatura, umidade, tempo de processamento, $\mathrm{pH}$, degradando facilmente devido a reações enzimáticas de óxido-redução (FREITAS et al., 2006; YUYAMA et al., 2008).

Desta maneira, a técnica de conservação adequada dos produtos é uma forma de se garantir sua estabilidade por mais tempo. Partindo desse pressuposto, o trabalho teve como objetivo realizar a desidratação de acerola em três temperaturas, e avaliar a estabilidade do produto a partir de análises físicas e químicas durante 75 dias de armazenamento.

\section{Material e métodos}

\subsection{Preparo e desidratação das amostras}

Os frutos de acerola 'Flor Branca' (Malpighia emarginata D.C.) foram colhidos no estádio de maturação maduro (100\% de coloração da casca vermelha) no primeiro semestre de 2015 em um pomar comercial localizado no Projeto Senador Nilo Coelho, Núcleo 4, Petrolina, PE (9²1'51.50"S e 40³9'43.80"W). Depois da colheita, os frutos foram levados em contentores plásticos aos Laboratórios de Armazenamento de Produtos Agrícolas e Química Geral da Universidade Federal do Vale do São Francisco, campus Juazeiro, onde foram submetidos à lavagem com água corrente e à sanitização em água clorada a $20 \mathrm{mg}$. $\mathrm{L}^{-1}$

Depois da sanitização, os frutos foram lavados novamente e dispostos em peneiras para secar em temperatura ambiente. Antes da desidratação, os frutos in natura foram avaliados quanto a: $\mathrm{pH}$, por meio de leitura em pHmetro digital modelo HI 221 (Splabor, São Paulo); sólidos solúveis (SS), determinados em Brix ${ }^{\circ}$ através de leitura em refratômetro digital modelo RTP-20ATC (Instrutherm, São Paulo); acidez titulável (AT), por meio da titulação do suco com 0,1 mol. $\mathrm{L}^{-1}$ de $\mathrm{NaOH}$ até $\mathrm{pH} 8,1$; umidade, através de secagem da amostra em estufa a $105^{\circ} \mathrm{C}$, até massa constante; Ácido ascórbico (AA), utilizando o método de Tillmans com titulação em 2-6 Diclorofenol- indofenol (DFI); cor, determinada através de leitura em colorímetro CR-400 Minolta (Konica Minolta, Japão) pelas escalas de cor Luminosidade (L), Croma (C) e ângulo hue (h), de acordo com Bezerra et al. (2011), Toebe et al. (2011). Todas as análises seguiram as metodologias preconizadas pelo Instituto Adolfo Lutz (IAL, 2008)

Depois da avaliação inicial, os frutos restantes foram divididos em dois lotes para a obtenção de frutos com e sem sementes, os quais foram submetidos à desidratação em Estufa de Circulação e Renovação de ar, modelo SL 102 (SOLAB, São Paulo), com capacidade para secagem de até $6 \mathrm{Kg}$, nas temperaturas de $60^{\circ} \mathrm{C}$, $70{ }^{\circ} \mathrm{C}$ e $80^{\circ} \mathrm{C}$. Para obtenção da polpa, as sementes foram retiradas manualmente, com o auxílio de uma faca, resultando em uma pasta homogênea. Para desidratação, as amostras de frutos, com semente e sem semente, foram dispostas em bandejas com capacidade para $1 \mathrm{~kg}$ de polpa. A desidratação foi realizada até os frutos atingirem umidade de aproximadamente 15\%, conforme recomendação de Celestino (2010). Para a análise inicial dos frutos in natura, assim como para cada temperatura de desidratação, foram utilizadas três repetições com 7 kg de frutos por repetição.

\subsection{Processamento da farinha}

Depois da desidratação, os frutos secos foram triturados em liquidificador, da marca Walita, 220 watts, durante 15 minutos, para obtenção da farinha. Posteriormente, 
as farinhas foram submetidas à caracterização inicial por meio de análises físicas e químicas de SS, pH, AT, AA e cor e, em seguida, dispostas em embalagens de plástico de tereftalato de polietileno, com tampa acoplada, e capacidade para $350 \mathrm{~mL}$.

O armazenamento ocorreu em temperatura de $25{ }^{\circ} \mathrm{C}$ e umidade relativa de $48 \%$. Durante 75 dias, as amostras foram submetidas a análises, a cada 15 dias, totalizando 6 tempos de condições de armazenamento (0, 15, 30, 45, 60 e 75 dias), com o objetivo de se avaliar o tempo de vida de prateleira. Os parâmetros avaliados seguiram as metodologias preconizadas pelo Instituto Adolfo Lutz (IAL, 2008).

O experimento foi conduzido em um delineamento inteiramente casualizado em esquema fatorial $3 \times 6$ (temperatura $x$ tempo). Para maior compreensão dos resultados, o esquema fatorial foi avaliado separadamente para a farinha de acerola com semente e farinha de acerola sem semente. Foram analisadas três temperaturas e o armazenamento, com seis tempos pré-definidos. A análise estatística foi realizada com o auxílio do programa ASSISTAT Versão 7.7 (SILVA; AZEVEDO, 2002), e os dados foram submetidos à análise de variância, comparando-se as médias por teste de Tukey a $5 \%$.

\section{Resultados e discussão}

$\mathrm{Na}$ Tabela 1, estão apresentados os resultados das análises físico-químicas obtidos para os frutos in natura, no momento da colheita. Os teores de AA no fruto in natura estão de acordo com a literatura, variando de 1,28\% a 0,97\%, conforme descrito por Fonseca et al. (2012) e Costa et al. (2011).

$\mathrm{Na}$ Tabela 2, estão os resultados para umidade das acerolas desidratadas. As temperaturas de $70^{\circ} \mathrm{C}$ e $80^{\circ} \mathrm{C}$ se mostraram com diferenças significativas entre 0 e 75 dias de armazenamento. Os teores de umidade mais elevados foram de $24,04 \%$ e $25,48 \%$, referentes ao produto resultante da secagem a $60{ }^{\circ} \mathrm{C}$ do fruto sem semente. Apesar dos valores elevados, as farinhas de acerola encontram-se dentro dos padrões estabelecidos pela ANVISA (BRASIL, 1978) para frutas desidratadas, que é de no máximo $25 \%$.

Gomes et al. (2004), ao estudarem estocagem da farinha de acerola a $25^{\circ} \mathrm{C}$ por 60 dias, comprovaram que, ao final, a umidade atingiu pouco mais de $8 \%$, havendo um acréscimo de $51,31 \%$ em relação ao teor inicial. Teores de $20,40 \%$ e $23,51 \%$ de umidade inicial e final, após armazenamento de 60 dias em condições ambientes, foram relatados por Alexandre et al. (2014) para farinha de pitanga acondicionada em embalagem flexível multifoliada (PET/PP) produzida a partir da secagem em estufa de circulação forçada a $70^{\circ} \mathrm{C}$.

Lisboa et al. (2012), ao acondicionarem farinha de figo-da-índia, desidratada a $90^{\circ} \mathrm{C}$ com estufa de circulação de ar forçado, verificaram um teor de água inicial de 7,5\%. Os autores armazenaram o pó à temperatura de $25^{\circ} \mathrm{C}$ e $40^{\circ} \mathrm{C}$ em embalagens compostas por camadas de filmes de plástico, alumínio e papel, com umidade controlada de $55 \%$ e $83 \%$.

A umidade é um parâmetro que deve ser mantido em baixo percentual, para evitar o aparecimento de microrganismos. De acordo com os dados encontrados na literatura, percebe-se que o tipo de embalagem utilizada influenciou diretamente o aumento da umidade do presente experimento, pois o material não propôs uma barreira suficiente para impedir a absorção de água do ar pelo produto.

De acordo com os resultados obtidos para a farinha, o pH foi superior ao determinado no fruto in natura mesmo durante o armazenamento (Tabela 3). Os valores de $\mathrm{pH}$ encontrados para a farinha de acerola, elaborada com e sem semente, ficaram abaixo de 4,5, valores esses que inibem a proliferação de muitos microrganismos (AQUINO et al., 2010). Nas amostras com semente, a temperatura de $70{ }^{\circ} \mathrm{C}$ foi a que apresentou maior valor de $\mathrm{pH}$ inicial, de 3,85, o qual diminuiu significativamente após os 75 dias de armazenamento. A farinha obtida de frutos com semente e desidratada a $60{ }^{\circ} \mathrm{C}$ mostrou-se mais ácida, com um valor de $\mathrm{pH}$ de 3,62, estando mais próximo do $\mathrm{pH}$ do fruto in natura, que também apresentou menor valor com relação às outras amostras.

Tabela 1. Características físico-químicas de acerola 'Flor Branca' in natura no momento da colheita.

\begin{tabular}{ccccc} 
pH & SS & $\begin{array}{c}\text { AT } \\
(\%)\end{array}$ & $\begin{array}{c}\text { AA } \\
(\%)\end{array}$ & $\begin{array}{c}\text { Umidade } \\
(\%)\end{array}$ \\
\hline 3,47 & 11 & 1,12 & 1,15 & 90,75 \\
\hline
\end{tabular}

Tabela 2. Médias de umidade (\%) para a acerola desidratada no estádio de maturação maduro com semente e sem semente para interação temperatura de secagem e tempo de armazenamento.

\begin{tabular}{|c|c|c|}
\hline \multicolumn{3}{|c|}{ Farinha com semente } \\
\hline \multicolumn{3}{|c|}{ Tempo de armazenamento (dias) } \\
\hline Temperatura $\left({ }^{\circ} \mathrm{C}\right)$ & 0 & 75 \\
\hline 60 & 21,69 Aa* & $22,17^{\mathrm{aA} *}$ \\
\hline 70 & $16,06^{\mathrm{bB} *}$ & $18,78^{\mathrm{bA}}$ \\
\hline 80 & $15,68^{\mathrm{bB} *}$ & $19,34^{\mathrm{bA}}$ \\
\hline \multicolumn{3}{|c|}{ Farinha sem semente } \\
\hline \multicolumn{3}{|c|}{ Tempo de armazenamento (dias) } \\
\hline Temperatura $\left({ }^{\circ} \mathrm{C}\right)$ & 0 & 75 \\
\hline 60 & $24,04^{\text {aA* }}$ & $25,48^{\mathrm{aA} *}$ \\
\hline 70 & $18,24^{\mathrm{bB} *}$ & $23,84^{\text {aA* }}$ \\
\hline 80 & $17,40^{\mathrm{bB} *}$ & $19,51^{\mathrm{bA}}$ \\
\hline
\end{tabular}

*As médias que apresentam as mesmas letras não diferem significativamente pelo teste de Tukey no nível de $5 \%$ de probabilidade. As letras maiúsculas comparam o tempo de armazenamento e as letras minúsculas, as temperaturas de desidratação. 
Produção e estabilidade de conservação de farinha de acerola desidratada em diferentes temperaturas

Reis, D. S. et al.

Tabela 3. pH de farinha de acerola 'Flor Branca' desidratada com ou sem semente no estádio de maturação maduro.

\begin{tabular}{|c|c|c|c|c|c|c|}
\hline \multicolumn{7}{|c|}{ Farinha com semente } \\
\hline \multicolumn{7}{|c|}{ Tempo de armazenamento (dias) } \\
\hline $\begin{array}{c}\text { Temperatura } \\
\left({ }^{\circ} \mathrm{C}\right)\end{array}$ & $\mathbf{0}$ & 15 & 30 & 45 & 60 & 75 \\
\hline 60 & $3,62^{\mathrm{CAB}}$ & $3,59^{\mathrm{bBC}}$ & $3,53^{\mathrm{bD}}$ & $3,55^{\mathrm{aCD}}$ & $3,60^{\mathrm{bAB}}$ & $3,64^{\mathrm{aA} *}$ \\
\hline 70 & $3,85^{\mathrm{aA} *}$ & $3,65^{\mathrm{aB}}$ & $3,64^{\mathrm{aB}}$ & $3,58^{\mathrm{ac}}$ & $3,64^{\mathrm{aB}}$ & $3,62^{\mathrm{aBC}}$ \\
\hline 80 & $3,74^{\mathrm{bA}}$ & $3,55^{\mathrm{cB}}$ & $3,56^{\mathrm{bB} *}$ & $3,49^{b C}$ & $3,55^{\mathrm{cB}}$ & $3,57^{\mathrm{bB} *}$ \\
\hline \multicolumn{7}{|c|}{ Farinha sem semente } \\
\hline \multicolumn{7}{|c|}{ Tempo de armazenamento (dias) } \\
\hline $\begin{array}{c}\text { Temperatura } \\
\left.\text { ( }{ }^{\circ} \mathrm{C}\right)\end{array}$ & $\mathbf{0}$ & 15 & 30 & 45 & 60 & 75 \\
\hline 60 & $3,88^{\mathrm{bA}}$ & $3,76^{a A^{*}}$ & $3,83^{a A *}$ & $3,86^{a A^{*}}$ & $3,89^{a A}$ * & $3,91^{\mathrm{aA} *}$ \\
\hline 70 & $4,16^{\mathrm{aA} *}$ & $3,92^{\mathrm{aAB}}$ & $3,89^{a B}$ & $3,86^{\mathrm{aB}}$ & $3,91^{\mathrm{AAB}}$ & $3,93^{\mathrm{aAB}}$ \\
\hline 80 & $4,18^{\mathrm{aA} *}$ & $3,80^{\mathrm{aB}}$ & $3,92^{\mathrm{aAB}}$ & $3,87^{\mathrm{aB}}$ & $3,91^{\mathrm{aB}}$ & $3,91^{\mathrm{aB}}$ \\
\hline
\end{tabular}

*As médias que apresentam as mesmas letras não diferem significativamente pelo teste de Tukey no nível de $5 \%$ de probabilidade. As letras maiúsculas comparam o tempo de armazenamento e as letras minúsculas, as temperaturas de desidratação.

Os resultados obtidos para farinha com semente indicam reduções no $\mathrm{pH}$ de $6 \%$ e $4,5 \%$, entre o primeiro e último dia, para as temperaturas de $70{ }^{\circ} \mathrm{C}$ e $80{ }^{\circ} \mathrm{C}$, respectivamente. Para a farinha desidratada a $60^{\circ} \mathrm{C}$, as alterações não foram significativas. Conforme Tonon et al. (2009), isso pode ser atribuído à menor temperatura de processo, que, apesar de demandar um maior tempo de desidratação, pode promover menor degradação dos açúcares e, consequentemente, resultar em maior semelhança quanto às características nutricionais do fruto in natura.

Os SS diminuíram entre o tempo inicial e final de armazenamento para as três temperaturas de desidratação avaliadas, em ambas as amostras analisadas (Tabela 4). No entanto, tal redução foi significativa apenas para a temperatura de $60{ }^{\circ} \mathrm{C}$ para farinha com semente, que também apresentou menor valor inicial de SS em relação às demais temperaturas de desidratação. Os resultados mostram que, apesar das diferenças entre os tempos de armazenamento, principalmente a partir dos 30 dias, tanto os açúcares como os outros teores avaliados tendem a se estabilizar.

Moura (2010), ao avaliar estabilidade em farinha de acerola armazenada, produzida a partir da polpa de frutos orgânicos de acerola verde, constatou diferenças significativas para os SS após 45 dias de armazenamento. De modo geral, a AT aumentou durante o armazenamento da farinha de acerola para praticamente todos os tratamentos (Tabela 5).

Este resultado era esperado, visto que houve redução concomitante do $\mathrm{pH}$ final, sendo que esses parâmetros físicos apresentam proporcionalidade inversa. Estudos realizados com farinha de acerola mostram valores estáveis
Tabela 4. Sólidos solúveis de farinha de acerola 'Flor Branca' desidratada com ou sem semente no estádio de maturação maduro

\begin{tabular}{|c|c|c|c|c|c|c|}
\hline \multicolumn{7}{|c|}{ Farinha com semente } \\
\hline \multicolumn{7}{|c|}{ Tempo de armazenamento (dias) } \\
\hline $\begin{array}{c}\text { Temperatura } \\
\left({ }^{\circ} \mathbf{C}\right)\end{array}$ & 0 & 15 & 30 & 45 & 60 & 75 \\
\hline 60 & $8,33^{\mathrm{bA}}$ & $7,33^{\mathrm{bAB}}$ & $6,33^{\mathrm{bB} *}$ & $8,0^{\mathrm{aAB}}$ & $8,00^{\mathrm{aAB}}$ & $8,20^{\mathrm{bAB}}$ \\
\hline 70 & $10,33^{\mathrm{aA} *}$ & $8,67^{\mathrm{abAB}}$ & $8,00^{\mathrm{aB}}$ & $7,83^{\mathrm{aB}}$ & $8,33^{\mathrm{aB}}$ & $9,17^{\mathrm{abAB}}$ \\
\hline 80 & $10,17^{\mathrm{aA} *}$ & $9,67^{\text {aA* }}$ & $8,33^{\mathrm{aAB}}$ & $7,33^{\mathrm{aB}}$ & $9,33^{a A *}$ & $9,77^{\mathrm{aA}}$ \\
\hline \multicolumn{7}{|c|}{ Farinha sem semente } \\
\hline \multicolumn{7}{|c|}{ Tempo de armazenamento (dias) } \\
\hline $\begin{array}{c}\text { Temperatura } \\
\left({ }^{\circ} \mathrm{C}\right)\end{array}$ & $\mathbf{0}$ & 15 & 30 & 45 & 60 & 75 \\
\hline 60 & $10,00^{\mathrm{aAB}}$ & $8,33^{\mathrm{cC}}$ & $8,67^{\mathrm{aBC}}$ & $7,97^{\mathrm{ac}}$ & $10,67^{\mathrm{aA} *}$ & $10,00^{\mathrm{AB}}$ \\
\hline 70 & $11,00^{\mathrm{aAB}}$ & $11,33^{\mathrm{aA} *}$ & $9,00^{\mathrm{aC}}$ & $9,00^{\mathrm{ac}}$ & $10,67^{\mathrm{aAB}}$ & $9,67^{\mathrm{aBC}}$ \\
\hline 80 & $11,17^{\text {aA* }}$ & $9,67^{\mathrm{bAB}}$ & $9,67^{\mathrm{a} A \mathrm{~B}}$ & $8,67^{\mathrm{aB}}$ & $9,60^{\mathrm{aAB}}$ & $9,67^{\mathrm{aAB}}$ \\
\hline
\end{tabular}

*As médias que apresentam as mesmas letras não diferem significativamente pelo teste de Tukey no nível de $5 \%$ de probabilidade. As letras maiúsculas comparam o tempo de armazenamento e as letras minúsculas, as temperaturas de desidratação.

Tabela 5. Acidez titulável de farinha de acerola 'Flor Branca' desidratada com ou sem semente no estádio de maturação maduro.

\begin{tabular}{|c|c|c|c|c|c|c|}
\hline \multicolumn{7}{|c|}{ Farinha com semente (\% ácido málico) } \\
\hline \multicolumn{7}{|c|}{ Tempo de armazenamento (dias) } \\
\hline $\begin{array}{c}\text { Temperatura } \\
\left({ }^{\circ} \mathbf{C}\right)\end{array}$ & $\mathbf{0}$ & 15 & 30 & 45 & 60 & 75 \\
\hline 60 & $6,01^{\mathrm{aC}}$ & $6,38^{\mathrm{bBC}}$ & $8,26^{\mathrm{aA}}$ & $7,74^{\mathrm{bAB}}$ & $7,95^{\mathrm{bAB}}$ & $8,15^{\mathrm{aA*}}$ \\
\hline 70 & $5,52^{\mathrm{aB}}$ & $8,55^{\mathrm{aA} *}$ & $8,47^{a A^{*}}$ & $9,12^{\mathrm{aA} *}$ & $8,05^{\mathrm{bA}}$ & $8,47^{\mathrm{aA} *}$ \\
\hline 80 & $4,99^{\mathrm{aB}}$ & $9,81^{\text {aA* }}$ & $6,48^{\mathrm{bB} *}$ & $10,27^{\mathrm{aA} *}$ & $9,46^{\mathrm{aA} *}$ & $9,48^{\mathrm{aA} *}$ \\
\hline \multicolumn{7}{|c|}{ Farinha sem semente (\% ácido málico) } \\
\hline \multicolumn{7}{|c|}{ Tempo de armazenamento (dias) } \\
\hline $\begin{array}{c}\text { Temperatura } \\
\left({ }^{\circ} \mathbf{C}\right)\end{array}$ & 0 & 15 & 30 & 45 & 60 & 75 \\
\hline 60 & $8,11^{\mathrm{aB}}$ & $9,59^{\mathrm{bA}}$ & $9,76^{\mathrm{A}^{*}}$ & $9,67^{\mathrm{bA}}$ & $9,20^{\mathrm{bA}}$ & $7,63^{\mathrm{cB}}$ \\
\hline 70 & $4,78^{b c}$ & $10,32^{\mathrm{abA}}$ & $9,68^{\mathrm{aAB}}$ & $10,48^{\mathrm{abA}}$ & $8,84^{\mathrm{bB} *}$ & $8,94^{\mathrm{bB} *}$ \\
\hline 80 & $4,12^{b c}$ & $10,63^{\mathrm{aA} *}$ & $8,66^{\mathrm{bB} *}$ & $10,75^{\mathrm{aA} *}$ & $10,25^{\mathrm{aA} *}$ & $10,18^{\mathrm{aA}}$ \\
\hline
\end{tabular}

*As médias que apresentam as mesmas letras não diferem significativamente pelo teste de Tukey no nível de $5 \%$ de probabilidade. As letras maiúsculas comparam o tempo de armazenamento e as letras minúsculas, as temperaturas de desidratação.

de $\mathrm{pH}$ durante o armazenamento (GOMES et al., 2004), sugerindo que mudanças químicas podem ter ocorrido no presente estudo durante o armazenamento de farinhas desidratadas a $70^{\circ} \mathrm{C}$ e $80^{\circ} \mathrm{C}$.

Os resultados da avaliação do AA na farinha de acerola das amostras, com e sem semente, estão discriminadas na Tabela 6. Comparando com o fruto in natura, a perda de água nos frutos durante a desidratação tende a concentrar os nutrientes. Verificou-se que as amostras sem semente tiveram, aos 0 dias de armazenamento, um conteúdo de AA até 19 vezes superior ao teor do fruto antes de ser desidratado, com concentrações de 19,45\%, 9,341\% e 
Produção e estabilidade de conservação de farinha de acerola desidratada em diferentes temperaturas

Reis, D. S. et al.

$10,29 \%$ para as temperaturas de $60{ }^{\circ} \mathrm{C}, 70^{\circ} \mathrm{C}$ e $80^{\circ} \mathrm{C}$, respectivamente.

Aquino et al. (2010), ao desidratar resíduo de acerola em secador elétrico com recirculação de ar forçado, obteve um teor de AA de 9,55\%, similar ao obtido no presente estudo para a farinha desidratada a $70{ }^{\circ} \mathrm{C}$. Valores menores foram determinados por Braga et al. (2011) quando caracterizaram farinhas de acerola produzidas a partir de resíduo industrial, relatando valores médios de $2,57 \%$ de AA. A eficiência da desidratação na perda de água do fruto, e consequente acúmulo de vitamina $\mathrm{C}$, tem sido observada também em caracterização nutricional de polpa de manga Rosa desidratada. Bezerra et al. (2011) constataram um aumento significativo de AA, na ordem de $47 \%$, no pó de manga, quando comparado com o teor inicial antes do processamento.

A temperatura é um dos fatores importantes para a conservação dos alimentos após a secagem, a exposição da matéria-prima a altas temperaturas e longos tempos de secagem promove alterações nas moléculas dos alimentos levando a processos oxidativos, como foi o caso do AA na farinha de acerola analisada (AQUINO et al., 2009).

Segundo Menezes et al. (2009), as perdas de AA são atribuídas também ao tipo de embalagem utilizada no produto. Os autores encontraram teores diferenciados do encontrado no presente trabalho ao verificar perda significativa de AA. Os valores inicial e final foram de 9,88\% e 4,98\% ao avaliar o comportamento físico-químico de acerola desidratada em estufa de circulação de ar durante 6 meses.

Maior índice de conservação do ácido foi observado para a farinha obtida no presente estudo com a desidratação a $70{ }^{\circ} \mathrm{C}$, após 75 dias de armazenagem. Observou-se uma diminuição de 23\% e 55\% para a farinha com semente e sem semente. A temperatura de secagem de $60{ }^{\circ} \mathrm{C}$ resultou na maior perda de $\mathrm{AA}$ durante o armazenamento, possivelmente devido ao maior tempo de secagem, o que resultou em maior oxidação de AA (Tabela 6).

Conforme a Tabela 7, a farinha com semente obtida à temperatura de $60{ }^{\circ} \mathrm{C}$ mostrou o menor valor do parâmetro L* (Luminosidade) da cor após o processamento. Com relação ao tempo de armazenamento, as amostras com semente desidratadas a $80{ }^{\circ} \mathrm{C}$ foram as únicas que não mostraram valores decrescentes com o tempo. Os valores de Luminosidade $\left(L^{*}\right)$ variam de 0 a 100 , sendo escura próxima de 0 e branca próxima de 100. As médias descritas na Tabela 7 demonstram que as amostras estão na faixa de 30 a 42, classificando-as com uma aparência de cor escura. Essa cor tendeu a um tom mais escuro no decorrer dos 75 dias de armazenamento.

Figueiredo et al. (2005) também observaram redução da luminosidade com o tempo de armazenamento de acerola em pó em diferentes condições de ambiente controladas.
Tabela 6. Concentração de ácido ascórbico em farinha de acerola 'Flor Branca' desidratada com ou sem semente no estádio de maturação maduro.

\begin{tabular}{|c|c|c|c|c|c|c|}
\hline \multicolumn{7}{|c|}{ Farinha com semente (\%) } \\
\hline \multicolumn{7}{|c|}{ Tempo de armazenamento (dias) } \\
\hline $\begin{array}{c}\text { Temperatura } \\
\left({ }^{\circ} \mathbf{C}\right)\end{array}$ & 0 & 15 & 30 & 45 & 60 & 75 \\
\hline 60 & $5,35^{\mathrm{aA} *}$ & $2,03^{\mathrm{cB}}$ & $1,96^{\mathrm{cB}}$ & $1,72^{\mathrm{cB}}$ & $1,49^{c B}$ & $1,27^{\mathrm{bB} *}$ \\
\hline 70 & $4,22^{\mathrm{bA}}$ & $3,54^{\mathrm{bA}}$ & $3,38^{\mathrm{bA}}$ & $3,51^{\mathrm{bA}}$ & $3,45^{\mathrm{bA}}$ & $3,21^{\mathrm{aA} *}$ \\
\hline 80 & $5,25^{a A^{*}}$ & $5,19^{\mathrm{aA} *}$ & $4,89^{\mathrm{aA} *}$ & $4,85^{\mathrm{aA} *}$ & $5,00^{\mathrm{aA} *}$ & $3,26^{\mathrm{aB}}$ \\
\hline \multicolumn{7}{|c|}{ Farinha sem semente (\%) } \\
\hline \multicolumn{7}{|c|}{ Tempo de armazenamento (dias) } \\
\hline $\begin{array}{c}\text { Temperatura } \\
\left({ }^{\circ} \mathbf{C}\right)\end{array}$ & 0 & 15 & 30 & 45 & 60 & 75 \\
\hline 60 & $19,45^{\text {aA* }}$ & $7,82^{\mathrm{aB}}$ & $7,41^{\mathrm{aB}}$ & $7,21^{\mathrm{aB}}$ & $5,57^{\mathrm{bc}}$ & $3,90^{\mathrm{aD}}$ \\
\hline 70 & $9,34^{\mathrm{bA}}$ & $8,87^{\mathrm{aAB}}$ & $7,30^{\mathrm{aBC}}$ & $7,62^{\mathrm{aB}}$ & $7,18^{\mathrm{ac}}$ & $4,18^{\mathrm{aD}}$ \\
\hline 80 & $10,29^{b A}$ & $8,64^{\mathrm{aB}}$ & $7,90^{\mathrm{aB}}$ & $7,41^{\mathrm{aB}}$ & $7,62^{\mathrm{aB}}$ & $3,54^{\mathrm{ac}}$ \\
\hline
\end{tabular}

*As médias que apresentam as mesmas letras não diferem significativamente pelo teste de Tukey no nível de 5\% de probabilidade. As letras maiúsculas comparam o tempo de armazenamento e as letras minúsculas, as temperaturas de desidratação.

Tabela 7. Luminosidade $\left(\mathrm{L}^{*}\right)$ de farinha de acerola 'Flor Branca' desidratada no estádio de maturação maduro com ou sem semente.

\begin{tabular}{|c|c|c|c|c|c|c|}
\hline \multicolumn{7}{|c|}{ Farinha com semente } \\
\hline \multicolumn{7}{|c|}{ Tempo de armazenamento (dias) } \\
\hline $\begin{array}{c}\text { Temperatura } \\
\left({ }^{\circ} \mathbf{C}\right)\end{array}$ & $\mathbf{0}$ & 15 & 30 & 45 & 60 & 75 \\
\hline 60 & $36,1^{\mathrm{bA}}$ & $35,7^{\mathrm{bAB}}$ & $33,7^{\mathrm{CAB}}$ & $33,5^{\mathrm{CBC}}$ & $31,0^{\mathrm{CCD}}$ & $30,3^{\mathrm{cD}}$ \\
\hline 70 & $39,9^{\mathrm{aAB}}$ & $40,4^{\mathrm{aAB}}$ & $41,7^{\mathrm{bA}}$ & $40,2^{\mathrm{bAB}}$ & $39,1^{\mathrm{bB} *}$ & $39,2^{\mathrm{bB} *}$ \\
\hline 80 & $40,9^{a c}$ & $41,8^{\mathrm{aABC}}$ & $44,1^{\text {aA* }}$ & $43,6^{\mathrm{AB}}$ & $43,1^{\mathrm{aABC}}$ & $41,3^{\mathrm{aBC}}$ \\
\hline \multicolumn{7}{|c|}{ Farinha sem semente } \\
\hline \multicolumn{7}{|c|}{ Tempo de armazenamento (dias) } \\
\hline $\begin{array}{c}\text { Temperatura } \\
\left({ }^{\circ} \mathbf{C}\right)\end{array}$ & 0 & 15 & 30 & 45 & 60 & 75 \\
\hline 60 & $38,1^{\mathrm{aA} *}$ & $38,0^{\mathrm{aAB}}$ & $38,6^{\mathrm{aA} *}$ & $37,9^{\mathrm{AAB}}$ & $37,4^{\mathrm{AB}}$ & $34,1^{\mathrm{aB}}$ \\
\hline 70 & $37,7^{\mathrm{aABC}}$ & $39,3^{\mathrm{aAB}}$ & $41,3^{\mathrm{aA} *}$ & $36,7^{\mathrm{aBC}}$ & $35,0^{\mathrm{ac}}$ & $35,5^{\mathrm{aBC}}$ \\
\hline 80 & $40,2^{\mathrm{aA} *}$ & $36,7^{\mathrm{aAB}}$ & $33,4^{\mathrm{bBC}}$ & $32,2^{\mathrm{bCD}}$ & $29,9^{b C D}$ & $29,2^{b D}$ \\
\hline
\end{tabular}

*As médias que apresentam as mesmas letras não diferem significativamente pelo teste de Tukey no nível de $5 \%$ de probabilidade. As letras maiúsculas comparam o tempo de armazenamento e as letras minúsculas, as temperaturas de desidratação.

De acordo com a Tabela 8, as farinhas de acerola sem semente e com semente apresentaram diminuição significativa do índice de croma das temperaturas de $60^{\circ} \mathrm{C}$ e $80^{\circ} \mathrm{C}$ em função do tempo de armazenamento. De acordo com a estatística, a intensidade de cor da fruta e polpa desidratada a $70^{\circ} \mathrm{C}$ permaneceu estável no decorrer e tempo final. A farinha da amostra sem semente desidratada apresentou os maiores valores de croma.

O croma é uma variável relacionada ao grau de pureza da cor, que delimita a intensidade de saturação pela escala de branco ou preto que a contém. Visualmente 
Produção e estabilidade de conservação de farinha de acerola desidratada em diferentes temperaturas

Reis, D. S. et al.

Tabela 8. Croma $\left(\mathrm{C}^{\star}\right)$ de farinha de acerola 'Flor Branca' desidratada no estádio de maturação maduro com ou sem semente.

\begin{tabular}{|c|c|c|c|c|c|c|}
\hline \multicolumn{7}{|c|}{ Farinha com semente } \\
\hline \multicolumn{7}{|c|}{ Tempo de armazenamento (dias) } \\
\hline $\begin{array}{c}\text { Temperatura } \\
\left({ }^{\circ} \mathrm{C}\right)\end{array}$ & 0 & 15 & 30 & 45 & 60 & 75 \\
\hline 60 & $34,1^{\mathrm{abA}}$ & $32,8^{\mathrm{abA}}$ & $33,2^{a A^{*}}$ & $32,7^{\mathrm{aA}}$ & $32,3^{a A^{*}}$ & $33,4^{a A^{*}}$ \\
\hline 70 & $36,3^{\mathrm{aA}}$ & $33,6^{a A^{*}}$ & $34,8^{a A^{*}}$ & $34,8^{a A^{*}}$ & $34,4^{a A^{*}}$ & $34,9^{a A^{*}}$ \\
\hline 80 & $32,0^{\mathrm{bA}}$ & $30,6^{\mathrm{bAB}}$ & $28,8^{\mathrm{bBC}}$ & $28,1^{\mathrm{bBC}}$ & $27,7^{\mathrm{bBC}}$ & $27,4^{\mathrm{bc}}$ \\
\hline \multicolumn{7}{|c|}{ Farinha sem semente } \\
\hline \multicolumn{7}{|c|}{ Tempo de armazenamento (dias) } \\
\hline $\begin{array}{c}\text { Temperatura } \\
\left({ }^{\circ} \mathbf{C}\right)\end{array}$ & $\mathbf{0}$ & 15 & 30 & 45 & 60 & 75 \\
\hline 60 & $35,6^{A b c}$ & $40,6^{\mathrm{aA}^{*}}$ & $36,1^{\mathrm{aB}}$ & $35,4^{\mathrm{aBCD}}$ & $33,0^{\mathrm{bCD}}$ & $32,4^{\mathrm{bD}}$ \\
\hline 70 & $37,1^{\mathrm{AA}^{*}}$ & $37,4^{\mathrm{bAB}}$ & $37,2^{\mathrm{aA}^{*}}$ & $37,3^{\mathrm{aA}^{*}}$ & $37,2^{\mathrm{aA}^{*}}$ & $38,0^{a^{*}}$ \\
\hline 80 & $34,9^{\mathrm{aB}}$ & $37,4^{\mathrm{bAB}}$ & $36,6^{\mathrm{aAB}}$ & $35,0^{\mathrm{aB}}$ & $34,5^{\mathrm{bB}} \mathrm{B}^{*}$ & $39,1^{\mathrm{aA}^{*}}$ \\
\hline
\end{tabular}

*As médias que apresentam as mesmas letras não diferem significativamente pelo teste de Tukey no nível de $5 \%$ de probabilidade. As letras maiúsculas comparam o tempo de armazenamento e as letras minúsculas, as temperaturas de desidratação.

Tabela 9. Ângulo hue (h) de farinha de acerola 'Flor Branca' desidratada com ou sem semente no estádio de maturação maduro.

\begin{tabular}{|c|c|c|c|c|c|c|}
\hline \multicolumn{7}{|c|}{ Farinha com semente } \\
\hline \multicolumn{7}{|c|}{ Tempo de armazenamento (dias) } \\
\hline $\begin{array}{c}\text { Temperatura } \\
\left({ }^{\circ} \mathbf{C}\right)\end{array}$ & 0 & 15 & 30 & 45 & 60 & 75 \\
\hline 60 & $67,3^{\mathrm{bA}}$ & $66,5^{\mathrm{bAB}}$ & $65,9^{\mathrm{bBC}}$ & $65,4^{\mathrm{bCD}}$ & $64,3^{\mathrm{bDE}}$ & $63,9^{\mathrm{bE}}$ \\
\hline 70 & $68,5^{\text {aA* }}$ & $68,3^{\mathrm{aA} *}$ & $67,6^{\mathrm{aAB}}$ & $68,0^{\mathrm{aAB}}$ & $66,7^{\mathrm{ac}}$ & $66,8^{\mathrm{aBC}}$ \\
\hline 80 & $68,1^{\mathrm{abAB}}$ & $68,5^{\mathrm{aA} *}$ & $68,2^{\mathrm{aAB}}$ & $67,0^{\mathrm{aCD}}$ & $67,3^{\mathrm{aBC}}$ & $66,1^{\mathrm{aD}}$ \\
\hline \multicolumn{7}{|c|}{ Farinha sem semente } \\
\hline \multicolumn{7}{|c|}{ Tempo de armazenamento (dias) } \\
\hline $\begin{array}{c}\text { Temperatura } \\
\left({ }^{\circ} \mathbf{C}\right)\end{array}$ & 0 & 15 & 30 & 45 & 60 & 75 \\
\hline 60 & $64,8^{\mathrm{aAB}}$ & $65,3^{\mathrm{abA}}$ & $65,9^{a A}$ & $62,5^{\mathrm{bBC}}$ & $62,5^{\mathrm{bBC}}$ & $61,0^{\mathrm{bc}}$ \\
\hline 70 & $65,1^{\mathrm{aA} *}$ & $66,2^{\mathrm{aA} *}$ & $66,3^{\mathrm{aA} *}$ & $67,2^{\mathrm{aA} *}$ & $66,8^{\mathrm{aA} *}$ & $64,2^{\mathrm{aA} *}$ \\
\hline 80 & $63,7^{\text {aA* }}$ & $63,6^{\mathrm{bA}}$ & $64,5^{\mathrm{aA} *}$ & $63,5^{\mathrm{bA}}$ & $62,9^{\mathrm{bA}}$ & $64,2^{\mathrm{aA}}$ \\
\hline
\end{tabular}

*As médias que apresentam as mesmas letras não diferem significativamente pelo teste de Tukey no nível de $5 \%$ de probabilidade. As letras maiúsculas comparam o tempo de armazenamento e as letras minúsculas, as temperaturas de desidratação.

as amostras se apresentaram com uma cor mais escura que tendeu à coloração vermelha. Esse fator pode ser atribuído à oxidação da AA durante a desidratação e armazenamento. O AA é um composto muito sensível quando exposto a altas temperaturas, e o escurecimento dos alimentos é o primeiro indicativo da sua degradação (TORALLES et al., 2008).

Houve diferenças significativas no ângulo hue (h) para ambos os tratamentos apenas na temperatura de $60{ }^{\circ} \mathrm{C}$ para a farinha de polpa desidratada durante a armazenagem. Na farinha sem semente, comportamento similar foi observado até 45 dias. A tonalidade das amostras decresce com o tempo, o que denota visualmente seu escurecimento, principalmente nas amostras de farinha sem semente desidratada a $60{ }^{\circ} \mathrm{C}$ que apresentaram um ângulo de 61,08 após 75 dias de armazenamento (Tabela 9).

O ângulo hue (h) caracteriza diferentes tonalidades de cor a cada $90^{\circ}$, sendo de $0^{\circ}$ para o vermelho, $90^{\circ}$ para o amarelo, $180^{\circ}$ para o verde e $270^{\circ}$ para o azul (SILVA et al., 2015). Os ângulos obtidos a partir da leitura com o colorímetro variam de $60^{\circ}$ a $69^{\circ}$. Evidenciam uma cor amarelo-vermelho para todas as amostras, sendo que a farinha da polpa se mostrou com tom mais amarelado. A farinha obtida com a desidratação a $60^{\circ} \mathrm{C}$ apresentou ângulos mais baixos, consequentemente foi a mais escura visivelmente, devido ao maior tempo de desidratação.

\section{Conclusão}

A desidratação de acerola pode ser realizada tanto com frutos sem semente como com semente, dependendo das necessidades locais e do produtor. Para todas as temperaturas de desidratação, os parâmetros de $\mathrm{pH}$ e teores de sólidos solúveis se mostraram semelhantes. Para as umidades finais obtidas e condições de acondicionamento realizadas, a Acidez Titulável aumentou em todas as amostras ao longo do armazenamento. Os resultados de cor indicam uma tendência para escurecimento da farinha obtida, principalmente para as amostras sem semente desidratadas a $60{ }^{\circ} \mathrm{C}$.

As farinhas processadas de frutos sem sementes apresentaram valores mais estáveis de $\mathrm{pH}$ e SS durante o armazenamento. Houve maior conservação do ácido ascórbico ao final do armazenamento para a farinha obtida com semente na temperatura de processo de $70{ }^{\circ} \mathrm{C}$. Pode-se dizer, portanto, que nestas condições houve melhor retenção das características nutricionais dessa fruta, bem como maior estabilidade no armazenamento.

O processamento da farinha de acerola revela uma opção para industrialização dessa fruta, considerada como fonte de ácido ascórbico resultando em um bom aproveitamento das suas qualidades nutricionais.

\section{Referências}

ALEXANDRE, H. V.; DE FIGUEIRÊDO, R. M. F.; QUEIROZ, A. J. M.; DE OLIVEIRA, E. N. A. Armazenamento de pitanga em pó. Comunicata Scientiae, Bom Jesus, v. 5, n. 1, p. 83-91, 2014.

AQUINO, A. C. M. S.; MOES, R. S.; LEÃO, K. M. M.; FIGUEIREDO, A. V. D.; CASTRO, A. A. Avaliação físico-química e aceitação sensorial de biscoitos tipo cookies elaborados com farinha de resíduos de acerola. Revista do Instituto Adolfo Lutz, São Paulo, v. 69, n. 3, p. 379-386, 2010.

AQUINO, L. P.; FERRUA, F. Q.; BORGES, S. V.; ANTONIASSI, R.; CORREA, J. L. G.; CIRILLO, M. A. Influência da secagem do pequi (Caryocar brasiliense Camb.) na qualidade do óleo extraído. Ciência Tecnologia de Alimentos, Campinas, v. 
Produção e estabilidade de conservação de farinha de acerola desidratada em diferentes temperaturas Reis, D. S. et al.

29, n. 2, p. 354-357, 2009. http://dx.doi.org/10.1590/S010120612009000200018.

BEZERRA, T. S.; COSTA, J. M. C.; AFONSO, M. R. A.; MAIA, G. A.; CLEMENTE, E. Avaliação físico-química e aplicação de modelos matemáticos na predição do comportamento de polpas de manga desidratadas em pó. Revista Ceres, Viçosa, v. 58, n. 3, p. 278-283, 2011

BRAGA, A. C. D.; LIMA, M. S.; AZEVEDO, L. C.; RAMOS, M. E. C. Caracterização e obtenção de farinha do resíduo gerado no processo industrial de clarificação do suco de acerola. Revista Semiárido de Visu, Petrolina, v. 1, n. 2, p. 126-133, 2011.

BRASIL. Agência Nacional de Vigilância Sanitária. Resolução CNNPA n 12, de 1978. Bombons e similares. Diário Oficial [da] República Federativa do Brasil, Brasília, DF, 24 jul. 1978. Disponível em: <http://www.anvisa.gov.br>. Acesso em: 20 maio de 2015.

CARNEIRO, T. B.; MELLO, J. G. Frutos e polpa desidratada Buriti (Mauritia flexuosa L.): aspectos físicos, químicos e tecnológicos. Revista Verde de Agroecologia e Desenvolvimento Sustentável Pombal, Paraíba, v. 6, n. 2, p. 105-111, 2011.

CELESTINO, C. M. S. Princípio de secagem de alimentos. Planaltina: Embrapa Cerrados, 2010. 51 p. (Circular Técnica 276).

COSTA, A. C. S.; LIMA, M. A. C.; ALVES, R. E. Caracterização físicoquímica de acerola e dos resíduos do processamento em dois estádios de maturação. In: SIMPÓSIO BRASILEIRO DE PÓS-COLHEITA DE FRUTAS, HORTALIÇAS E FLORES, 2011, Nova Friburgo. Anais... Rio de Janeiro: Embrapa Agroindústria Tropical, 2011.

FIGUEIREDO, R. M. F.; QUEIROZ, A. J. M.; MARTUCCI, E. T. Alterações de cor da acerola em pó sob condições controladas. Revista Brasileira de Produtos Agroindustriais, Campina Grande, v. 7, n. 1, p. 49-57, 2005. http://dx.doi.org/10.15871/15178595/rbpa.v7n1p49-57.

FONSECA, M. D.; TEIXEIRA, B. V. F.; SILVA, L. F. L.; AMDRADE, M. V. S.; REIS, R. C.; VIANA, E. D. S. Caracterização físico-química de híbridos de acerola desenvolvidos pela Embrapa mandioca e fruticultura. In: CONGRESSO BRASILEIRO DE CIÊNCIA E TECNOLOGIA DE ALIMENTOS, 23., 2012, Campinas. Anais... Campinas: SBCTA, 2012. 1 CD-ROM.

FREITAS, C.; MAIA, G. A.; COSTA, J. M. C. D.; FIGUEIREDO, R. W. D.; RODRIGUES, M. D. C. P.; SOUSA, P. H. M. D. Estabilidade do suco tropical de acerola (Malpighia emarginata DC) adoçado envasado pelos processos hot-fill e asséptico. Ciência e Tecnologia Alimentos, Campinas, v. 26, n. 3, p. 544-549, 2006. http://dx.doi.org/10.1590/S0101-20612006000300010.

GOMES, P. M. A.; FIGUEIRÊDO, R. M. F.; QUEIROZ, A. J. M. Armazenamento da polpa de acerola em pó a temperatura ambiente. Ciência e Tecnologia de Alimentos, Campinas, v. 24, n. 3, p. 384-389, 2004. http://dx.doi.org/10.1590/S010120612004000300014

INSTITUTO ADOLFO LUTZ - IAL. Métodos físico-químicos para análise de alimentos. São Paulo: IAL, 2008.
LISBOA, C. G. C.; FIGUEIRÊDO, R. M. F.; QUEIROZ, A. J. M. Armazenamento de figo-da-índia em pó. Revista Brasileira de Engenharia Agrícola e Ambiental, Campina Grande, v. 16, n. 2, p. 216-221, 2012. http://dx.doi.org/10.1590/S141543662012000200013.

MENDONÇA, V.; MEDEIROS, F. L. Culturas da aceroleira e do maracujazeiro. Mossoró: Universidade Federal Rural do Seminário, 2011. (Boletim Técnico; IV).

MENEZES, A. R. V.; SILVA JUNIOR, A.; CRUZ, H. L. L.; ARAUJO, D. R.; SAMPAIO, D. D. Estudo comparativo do pó da acerola verde (Malphigia emarginata DC) obtido em estufa por circulação de ar e por liofilização. Revista Brasileira de Produtos Agroindustriais, Campina Grande, v. 11, n. 1, p. 1-8, 2009. http://dx.doi.org/10.15871/1517-8595/rbpa.v11n1p1-8.

MOURA, M. S. Estabilidade de acerola em pó oriunda de cultivo orgânico. 2010. Dissertação (Mestrado em Ciência e Tecnologia de Alimentos)-Universidade Federal do Ceará, Fortaleza, 2010.

SEGTOWICK, E. C. S.; BRUNELLI, L. T.; VENTURINI FILHO, W. G. Avaliação físico-química e sensorial de fermentado de acerola. Brazilian Journal of Food Technology, Campinas, v. 16, n. 2, p. 147-154, 2013. http://dx.doi.org/10.1590/S198167232013005000015.

SILVA, D. F. P.; SIQUEIRA, D. L.; PEREIRA, C. S.; SALOMÃO, L. C. C.; STRUIVING, T. B. Caracterização de frutos de 15 cultivares de mangueira na Zona da Mata mineira. Ceres, Viçosa, v. 56, n. 6, p. 783-789, 2015.

SILVA, F. A. S.; AZEVEDO, C. A. V. Versão do programa computacional Assistat para o sistema operacional Windows. Revista Brasileira de Produtos Agroindustriais, Campina Grande, v. 4, n. 1, p. 71-78, 2002. http://dx.doi.org/10.15871/15178595/rbpa.v4n1p71-78.

TOEBE, M.; BOTH, V.; CARGNELUTTI FILHO, A.; BRACKMANN, A.; STORCK, L. Dimensionamento amostral para avaliar firmeza de polpa e cor da epiderme em pêssego e maçã. Revista Ciência Agronômica, Fortaleza, v. 42, n. 4, p. 1026-1035, 2011. http:// dx.doi.org/10.1590/S1806-66902011000400027

TONON, R. V.; BRABET, C.; HUBINGER, M. D. Influência da temperatura do ar de secagem e da concentração de agente carreador sobre as propriedades físico-químicas do suco de açaí em pó. Ciência e Tecnologia de Alimentos, Campinas, v. 29 , n. 2, p. 444-450, 2009. http://dx.doi.org/10.1590/S010120612009000200034.

TORALLES, R. P.; VENDRUSCOLO, J. L.; VENDRUSCOLO, C. T.; DEL PINO, F. A. B.; ANTUNES, P. L. Determinação das constantes cinéticas de degradação do ácido ascórbico em purê de pêssego: efeito da temperatura e concentração. Ciência e Tecnologia de Alimentos, Campinas, v. 28, n. 1, p. 18-23, 2008. http://dx.doi.org/10.1590/S0101-20612008000100004.

YUYAMA, L. K. O.; MAEDA, R. N.; PANTOJA, L.; AGUIAR, J. P. L.; MARINHO, H. A. Processamento e avaliação da vida-deprateleira do tucumã (Astrocaryum aculeatum Meyer) desidratado e pulverizado. Ciência e Tecnologia de Alimentos, Campinas, v. 28, n. 2, 408-412, 2008. 